\title{
The epidemic of the cesarean section in private hospital in Puebla, MÃ@xico
}

\begin{abstract}
The world health organization established that there are no valid justifications to perform more than $15 \%$ cesarean surgeries. The Mexican official norm establishes that cesarean section could be between $12-17 \%$.

Objective: Determine the real incidence of cesarean interventions in México and the factors associated with it.

Methods: Sociodemographic data's from 504 women attended in public and private hospitals in Puebla City were collected.

Results: There has been a significant, out of the norm limits, an increment in the cesarean surgery in México, reaching up to $57.3 \%$ of all deliveries, notably the cesarean section reaches up to $85.6 \%$ in private hospitals. We also found that the cesarean decision significantly correlates with the age, socioeconomic status and scholar mother's degree.

Conclusion: It is important to evaluate what is happening and the factors associated with these numbers in Mexico, and to take corrective measurements oriented to decrease the number of cesarean deliveries that implies a well-known risk for both the mother and the baby.
\end{abstract}

Keywords: age, education level, economic status, public or private health system, cesarean
Volume 2 Issue 6 - 2015

\author{
Elena Soto Vega, ${ }^{1,2}$ Urrutia-Osorio Marta,' \\ Arellano-Valdez Fernando,' Lopez Begines \\ lliana Yazmin,' Hernÿndez Romero Christian \\ Hazel' \\ 'Departamento de Ciencias de la Salud, Universidad Popular \\ Autónoma del Estado de Puebla, México \\ ${ }^{2}$ Centro de Investigación, Una Nueva Esperanza A.B.P, México
}

\begin{abstract}
Correspondence: Elena Soto Vega, Universidad Popular Aut noma del Estado de Puebla, 2 I sur I I03. Barrio de Santiago, C.P 72410, Puebla, México, Tel 52 (222) 2299400, ext. 7140,
\end{abstract} Email elena.soto0I@upaep.mx

Received: June 23, 2015 | Published: September 2, 2015

\section{Introduction}

The cesarean section is the most common major surgical procedure performed in the world. The World Health Organization has established that there are no valid justifications to perform cesarean section in more than a $10-15 \%{ }^{1}$ of the cases. As a matter of fact, the Mexican official norm (NOM-007-SSA2-1993) states as a correct ratio between $12-17 \%{ }^{2,3}$ However, nowadays cesarean section is being performed much more frequently, reaching high numbers worldwide. ${ }^{4}$ Until 2012, México was the country with higher number of cesarean section in the world, reaching a total of $45.2 \%{ }^{5}$ It is worth to note cesarean practice in the private medical practice reach up to $69.8 \% .^{5}$

The cesarean section poses short and long term health risk to the mother and infants. A cesarean section procedure has precise indications and these are divided in the elective and urgent indications. The elective ones are: placenta previa, two previous cesarean section (iterative cesarean section), maternal risk factors harmful to the fetus (HIV), cephalopelvic disproportion, breech presentation, transverse lie, among others. The urgent indications are: dystocia, fetal distress, cord prolapse, placental abruption, ruptured vasa and poor maternal status. $^{6}$

The cesarean section rate has become more prevalent over the years without a clear medical justification and regardless it is associated with an increase in morbidity and mortality of mothers and children. Cesarean is associated with adverse effects such as: maternal death, emergency hysterectomy, thromboembolic events, wound infections, reduced fertility, prolonged hospital stay with greater number of hospital readmissions, problems with physical recovery and chronic pelvic pain. ${ }^{7-9}$ Adverse effects to the newborns are less well documented; but the evidence suggests an increase of the respiratory distress syndrome after a cesarean. In addition, there are chronic diseases that occur more frequently in children born by a cesarean section such as: asthma, type 1 diabetes and allergic rhinitis. It also has to be taken into consideration the public costs in health that are made by these surgical procedures. A study by González Pérez et al. ${ }^{10}$ showed that the costs of the excessive number of cesarean section performed in Jalisco, México exceed several millions of dollars annually, having a significant effect on the economy. ${ }^{10}$

Regardless of these facts, cesarean section has some medical advantages over vaginal delivery like thee vasion of labor pain, the selection of delivery schedule, and finally the lower prevalence of urinary incontinence. Another maternal and obstetric factors that insides in the increase of the cesarean rate are the older maternal age, the greater weight gain during pregnancy, obesity and the higher prevalence of nulliparity. ${ }^{11,12}$

Although there are studies that attempt to explain the clinically and economically significant increase in cesarean section as seen above, we lack sufficient demographic studies that allow us to accurately define the factors that are contributing to increase the cesarean section in Mexico. With the aim to understand the causes of the cesarean section rate, we analyzed the influence of age, scholar degree, medical system (public-private) and economic income.

\section{Material and methods}

Data were collected from 5 different public and 3 private hospitals in Puebla, México, from November 2013 to February 2014. A total of 517 mothers agreed to participate; however, 13 were excluded (because of incorrect fulfill of survey). Of the 504 participants, 125 were from private hospitals and 379 from public hospitals.

Women were randomly selected and were interviewed immediately in the post-partum or post-cesarean period, during their 
stay in the hospital. The survey contemplates the following variables: mother age, gestational weeks, economic income, educational level, and the clinical variables were: the type of lie and presentation of the fetus, previous cesarean sections or vaginal births, if there were any complications during pregnancy or during the postpartal period, We also obtained data, from the medical history, about the decision making on the way of delivery method and also about who sets indication for cesarean section.

The data were analyzed using graph pad $6,2 \times 2$ contingency tables. Statistical differences between groups were done employing chi square test with Yate's correction. The relative risk (RR) and Odds ratio (OR) were calculated using the medcalc calculator with a confidence interval (CI) of $95 \%$. The $p$ value was statistically significant when $p$ was $\leq 0.05$. The protocol was approved by the Ethicesarean section Committee of the Universidad Popular Autónoma del Estado de Puebla, an informed consent was obtained from all the participants.

\section{Results}

The patient's age range was between 13 and 42 years old. The average age was $25.7 \pm 6.1$ years old; women attended in public hospitals were $24.7 \pm 6.2$ years old and those attended in private hospitals were $31.1 \pm 6.6$ years old, there was non-significant difference in age between groups. The data analysis showed that $57.3 \%$ of the women underwent cesarean section and $42.7 \%$ experienced vaginal birth. An important factor that should be taken into account in the choice between vaginal birth or cesarean section is the mother age, it is interesting that as mother's age increases the frequency of the vaginal birth decreases and the cesarean increases (Figure 1).

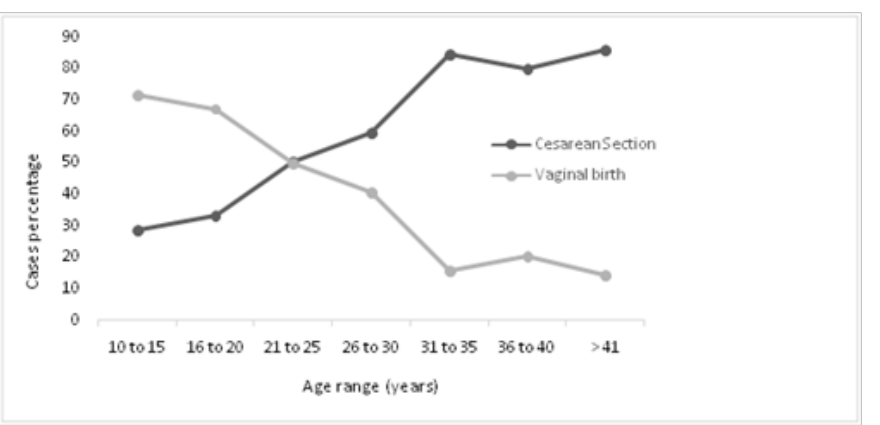

Figure I Relationship between the cumulative percentage of cesarean section (black line) or vaginal birth (gray line) realized according to the women age groups $(n=504)$. Birth frequency is clearly biased towards younger age.

The next point was to evaluate the percentage of cesareans and vaginal delivery according to the health system in which the mother was attended. The public system has a similar percentage of vaginal birth versus cesarean section ( $52.2 \%$ vs $47.8 \%$ ), while private hospitals have a significantly higher cesarean section percentage ( $14.4 \%$ vs 85.6 $\%)(p<0.00001)$. The difference in the cesarean section rate among public and private hospital is significantly different $(\mathrm{p}<0.0001)$, being the cesarean section much more common in private hospitals.

We divided the population in two categories: women older or younger than 30 years old and the result showed a significant difference in the delivery method election, the cesarean section is significantly increased in the women older than 30 years old $(\mathrm{p}<0.0001) \mathrm{RR}=0.58$ $(0.51-0.66), \mathrm{OR}=0.19(0.12-0.32)$. We also analyzed the risk of having cesarean section for those women attended in a private hospital, according to the age group (younger or older than 30 years) and we found a significant result $(\mathrm{p}=0.002)$ with a $\mathrm{RR}=0.43(0.32$
$0.58), \mathrm{OR}=0.08(0.02-0.32)$ these results are similar to the results of the entire group $(n=504)$ (Table 1). The gestational week's analysis showed no difference between women attended in public or private hospitals ( $38.3 \pm 2.75$ and $39.04 \pm 2.40$ respectively), dividing the group in younger or older than 30 there was no difference among gestational weeks.

Table I This table shows the percentage of women that underwent to a cesarean section (CS) or a vaginal birth (VB) in public or private system according to their age group (older or younger than 30 )

\begin{tabular}{lllll}
\hline Health system & \multicolumn{2}{l}{ Public (\%) } & \multicolumn{2}{l}{ Private (\%) } \\
\hline Delivery method & CS & VB & CS & VB \\
$<30$ & 38.26 & 49.44 & 33.06 & 12.9 \\
$>30$ & 6.42 & 5.86 & 53.22 & 0.8
\end{tabular}

The medical records were reviewed to know the causes that led the cesarean decision, it was found that in public system $42.5 \%$ were related to a medical emergency, meanwhile in private hospital only $16.7 \%$ were considered an emergency, the rest of the cesareans were programed, there are significant statistical differences between both systems in the frequency and the reason originating the cesarean $(p<0.0001)$. The most common indication for cesarean section was iterative cesarean and in this item mother's decision is the most frequent reason for the surgery (Table 2).

Table 2 Table of the percentage of the most common indications for cesarean surgery in private and public hospitals

\begin{tabular}{lll}
\hline Programmed cesarean section indication & $\begin{array}{l}\text { Private } \\
\text { hospital }\end{array}$ & $\begin{array}{l}\text { Public } \\
\text { hospitals }\end{array}$ \\
\hline Iterative Cesarean & $40.80 \%$ & $38.50 \%$ \\
Abnormal lie or Presentation & $13.20 \%$ & $28.80 \%$ \\
Alterations of the Placenta and Umbilical cord & $10.50 \%$ & $8.70 \%$ \\
Fetal Abnormalities & $9.20 \%$ & $3.80 \%$ \\
Maternal Disease & $10.50 \%$ & $9.60 \%$ \\
Multiple Pregnancies & $5.30 \%$ & $2.90 \%$ \\
\hline
\end{tabular}

Maternal scholar degree was considered as a factor associated with the election of the delivery method and it was found that $93.3 \%$ of the mothers with a $\mathrm{PhD}$ or a master elected a cesarean section (Table $3)$. We compared the group as professional women $(\mathrm{PhD}$, master or bachelors) and non-professional women (high, junior or elementary school), we found that the risk of cesarean increases significantly as the scholar degree increases $(\mathrm{p}<0.001) \mathrm{RR}=1.70(1.47-1.95) \mathrm{OR}=4.27$ (2.74-6.66). The Table 3 shows that the decision to be attended in a private hospital depends also of women scholar degree, those with $\mathrm{PhD}$, master's or Bachelor prefer to deliver in a private hospital, so this increase the cesarean risk.

The monthly family income was also evaluated and $45.1 \%$ of the sample population have a monthly income considered as extreme poverty ( $0-230$ USD per month) and only $10.7 \%$ earns over $\$ 1150$ USD considered high median or high income, all the women in this last rank were attended in a private hospital $(n=54)$. When compared the monthly income between women attended at a private hospital and a public hospital the difference was found to be extremely statistically significant $(\mathrm{p}<0.0001)$ (Table 4).

To evaluate if the economic income influences the decision of the delivery method, we divided the population according to the economic status in two groups: those with low income (low, medium, 
poverty or extreme poverty) and those with-high income (medium or high medium). The data indicate that there was a significant difference in the delivery method between both populations; in the group with a high income the cesarean section was significantly more frequent than in those considered as low income group $(\mathrm{p}<0.0001) \mathrm{RR}=0.54(0.48$ $0.6)$ and $\mathrm{OR}=0.07(0.03-0.17)$ and the difference also is reflected in the public health in which women is attended (Table 5).

Table 3 Relationship between scholarity and the decision of cesarean section in private and public hospitals

\begin{tabular}{|c|c|c|c|c|}
\hline \multirow{2}{*}{ Scholarity } & \multicolumn{2}{|c|}{$\begin{array}{l}\text { Private hospital } \\
(n=125)\end{array}$} & \multicolumn{2}{|c|}{$\begin{array}{l}\text { Public hospitals } \\
(n=379)\end{array}$} \\
\hline & Cesarean & $\begin{array}{l}\text { Vaginal } \\
\text { birth }\end{array}$ & Cesarean & $\begin{array}{l}\text { Vaginal } \\
\text { birth }\end{array}$ \\
\hline $\mathrm{PhD}$ & 100 & 0 & 0 & 0 \\
\hline Master's & 92.3 & 7.6 & 0 & 0 \\
\hline Bachelor & 56.7 & 6.7 & 18.3 & 18.3 \\
\hline High School & 6.7 & 4.4 & 43.4 & 45.6 \\
\hline Junior High & 1.5 & 0.7 & 40.3 & 57.5 \\
\hline Primary School & 0 & 0 & 56.6 & 43.4 \\
\hline None & 0 & 0 & 42.9 & 57.1 \\
\hline
\end{tabular}

Table 4 Difference in monthly family income of women that selected private or public hospitals, expressed in USD

\begin{tabular}{lll}
\hline Monthly family income & $\begin{array}{l}\text { Private } \\
\text { hospitals (\%) }\end{array}$ & $\begin{array}{l}\text { Public } \\
\text { hospitals (\%) }\end{array}$ \\
\hline Extreme Poverty $(\$ 0-230)$ & 5.6 & 56.5 \\
Poverty $(\$ 230-\$ 460)$ & 2 & 32.4 \\
Low Medium $(\$ 460-\$ 924)$ & 25.2 & 11.1 \\
Medium $(\$ 924-\$ 1150)$ & 23.2 & 0 \\
High Medium $(>\$ 1150)$ & 44 & 0 \\
Total & $100 \%$ & $100 \%$ \\
\hline
\end{tabular}

Table 5 Percentage of cesarean and vaginal birth according the income classification in private and public hospitals

\begin{tabular}{lllll}
\hline \multirow{2}{*}{ Economic status } & \multicolumn{2}{c}{ Private hospitals } & \multicolumn{2}{c}{ Public hospitals } \\
\cline { 2 - 5 } & Cesarean & $\begin{array}{l}\text { Vaginal } \\
\text { birth }\end{array}$ & Cesarean & $\begin{array}{l}\text { Vaginal } \\
\text { birth }\end{array}$ \\
\hline Extreme poverty & 0.59 & 0.79 & 17.85 & 25.19 \\
Poverty & 0.39 & 0 & 13.29 & 10.51 \\
Low Medium & 4.76 & 1.58 & 4.76 & 3.57 \\
Medium & 5.55 & 0.19 & 0 & 0 \\
High, Medium or High & 9.9 & 2.57 & 0 & 0 \\
\hline
\end{tabular}

\section{Discussion}

The prevalence in cesarean section has had a significant increase in the last years. In Mexico in 2012 the percentage of cesarean section was $45.2 \%$, however nowadays we found that it has risen up to $57.3 \%$, and in the case of private hospitals the percentage increases to an alarming number of $85.6 \%$, which is one of the highest ever found in any previous survey in all the world. ${ }^{5,13-18}$ It is obvious that the increase in the cesarean section rates has not been satisfactorily faced by the health sector authorities in México.
The women age seems to be directly correlated with the selection of the cesarean section, increasing dramatically in those older than 30 years old and generally the decision for cesarean is taken by the mothers without medical reason, this result agrees with several studies worldwide that also shows that the mother's age is important in this decision. ${ }^{13,19}$

The scholar degree seems to be very important in the selection of the delivery method; $93.3 \%$ of the mothers with a profession elected a cesarean. In China educated women were 3-4 times more likely to have a cesarean section as compared to illiterates. ${ }^{20}$ Women may consider cesarean as less painful, convenient and safer option than vaginal birth. ${ }^{21-24}$ Besides, the cesarean allows women to have certainty on the development of the birth, the modern medicine also contributes to the confidence of the most educated women, which is also an important factor to consider.

The result obtained with the analysis of the economic income is similar to other studies realized in countries like China, Brazil and Italy. ${ }^{20,25}$ It is understandable that women with a stable socioeconomic status prefers to be attended in the private sector to avoid all the administrative procedure in the public hospital that is also associated with a poor medical attention due to the quantity of women that each doctor has to attend daily.

The highest rate of cesarean section found in the private hospitals could be explained by the economic incentives to doctors, which are usually three times larger than those obtained with a vaginal birth, in a cesarean procedure in México a doctor could earn between $\$ 1000$ 2900 USD in the private sector. However, economic incentives do not explain what happens in the public hospitals where doctors do not receive extra payment for performing a cesarean section. Our results are similar to those registered in Ethiopia, they found 3 times higher levels of cesarean in the private compared to public sector. ${ }^{18}$

Due to the increment observed in recent years of the cesarean section in Mexico, it is very important to develop a formal line of research to define what is happening, and propose a national delivery health law that warrants women to be well informed of their options and make an informed choice of the delivery method they desire. It is important to take into account the economic cost for the public health system of the increase in the cesarean section which means several millions of dollars spent annually on unnecessarily health services.

\section{Acknowledgments}

To all the institutions that collaborated in this survey: Hospital General No 36 IMSS, Cruz Roja de México-Puebla, Hospital de la Mujer Puebla, Hospital Ángeles de Puebla, Hospital Puebla, Beneficencia Española-Puebla.

\section{Conflicts of interest}

The authors declare there is no conflict of interests.

\section{References}

1. Bettegowda VR, Dias T, Davidoff MJ, et al. The relationship between cesarean delivery and gestational age among us singleton births. Clin Perinatol Epidemiol. 2008;21(2):98-113.

2. SSA. Norma Official Mexicana, NOM 010-SSA2-2010. Women and new born care during pregnancy, childbirth and postpartum. Criteria and procedures for the services; 2010.

3. Gibbons, Belizán JM, Lauer JA, et al. The global numbers and costs of 
additionally needed and unnecessary caesarean sections performed per year overuse as a barrier to universal coverage. World health report; 2010 .

4. Rosado-Gorocica JD, Montoya-López S, Dzul-Rosado KR, et al Frequency, indications and knowledge of pregnant women about caesarean section: The case of a hospital in the city of Mérida, Yucatán. Rev Biomed. 2013;24:100-108.

5. Suárez-López L, Campero L, Vara-Salazar E, et al. Sociodemographic and reproductive characteristic cesarean section associated with increased cesarean in Mexico. Salud Pública Méx. 2013;55(Suppl 2):S225-S234.

6. Souzza JP, Gulmezoglu AM, Laopaiboon M, et al. Caesarean section without medical indications is associated with an increased risk of adverse short-term maternal outcomes: the 2004-2008 WHO Global survey on Maternal and Perinatal Health. BMC Med. 2010;8:71-80.

7. Declercq E, Barger M, Cabral HJ, et al. Maternal outcomes associated with planned primary cesarean births compared with planned vaginal births. Obstet Gynecol. 2007;109(3):669-677.

8. Declercq E, Cunningham DK, Johnson C, et al. Mothers' reports of postpartum pain associated with vaginal and cesarean deliveries: Results of a national survey. Birth. 2008;35(1):16-24.

9. Deneux-Tharaux C, Carmona E, Bouvier-Colle $\mathrm{MH}$, et al. Postpartum maternal mortality and cesarean delivery. Obstet Gynecol. 2006;108(3):541-548.

10. Gonzalez-Perez GJ, Vega-Lopez MG, Cabrera-Pivaral C, et al. Caesarean sections in Mexico: are there too many? Health Policy Plan. 2001;16(1):62-67.

11. Hourani M, Ziade F, Rajab M. Timing of planned caesarean section and the morbidities of the newborn. $N$ Am J Med. 2011;3(10):465-468.

12. Caughey AB, Sundaram V, Kaimal AJ, et al. Maternal and neonatal outcomes of elective induction of labor. Evid Rep Technol Assess (Full Rep). 2009;(176):1-257.

13. Gebremedhin S. Trend and socio-demographic differentials of caesarean section rate in Addis Ababa, Ethiopia: Analysis based on Ethiopia demographic and health surveys data. Reprod Health. 2014;11(1):14-20.
14. Henke R, Wier LM, Marder WD, et al. Geographic variation in cesarean delivery in the United States by payer. BMC Pregnancy Childbirth. 2014;14(1):387.

15. Henke RM, Marder WD, Friedman BS, et al. Geographic variation: a view from the hospital sector. Med Care Res Rev. 2011;68(6):699-711.

16. Salomón-Benítez MF, Tejeda-Rangel E, Osuna-Ramírez I. Caesarean sections in Mexico: Trends and Associated Factors literature review. Arch Salud Sin. 2013;8(1):30-36.

17. Penn Z, Ghaem-Maghami S. Indications for caesarean section. Best Pract Res Clin Obstet Gynaecol. 2001;15(1):1-15.

18. Charoenboon C, Srisupundit K. Rise in cesarean section rate over a 20 year period in a public sector hospital in northern Thailand. Arc Gynecol Obstet. 2013;287(1):47-52.

19. Betrán AP, Merialdi M, Lauer JA, et al. Rates of caesarean section: analysis of global, regional and national estimates. Paediatr Perinat Epidemiol. 2007;21(2):98-113.

20. Feng XL, Xu L, Guo Y, et al. Factors influencing rising Caesarean section rates in China between 1988 and 2008. Bull World Health Organ. 2012;90:30-39.

21. Mukherjee SN. Rising cesarean section rate. J Obstet Gynecol India. 2006;56(4):298-300.

22. Castro A. Commentary: increase in caesarean section may reflect medical control not women's choice. BMJ. 2001;319(7222):1401-1402.

23. Showalter E, Griffin A. Commentary: all women should have a choice. BMJ. 1999;319(7222):1401.

24. Hopkins K, Amaral E. The role of nonclinical factors in Caesarean section rates in Brazil. 2005;1-10.

25. Fesseha N, Getachew A, Hiluf M, et al. A national review of Caesarean delivery in Ethiopia. Int J Gynaecol Obstet. 2011;115(1):106-111. 